\title{
A Gap Waveguide Fed Circular Polarization Antennas in the Millimeter Wave Range
}

\author{
Dayan Pérez-Quintana ${ }^{1,2}$, Alicia Torres-García ${ }^{1,2}$, Iñigo Ederra ${ }^{1,2}$, Miguel Beruete ${ }^{1,2}$ \\ ${ }^{1}$ Department of Electrical, Electronic and Communications Engineering, Public University of Navarra, Spain \\ ${ }^{2}$ Institute of Smart Cities (ISC), Public University of Navarra, Navarra, Spain \\ dayan.perez@unavarra.es, aliciaelena.torres@unavarra.es, inigo.ederra@unavarra.es, miguel.beruete@unavarra.es
}

\begin{abstract}
In this work, a novel circular polarization (CP) antennas in ridge gap waveguide (RGW) working in the $\mathrm{V}$ band of the millimeter-wave spectrum is presented. $\mathrm{CP}$ is generated in a simple and effective way by means of two orthogonal feeder arms that excite a $\mathrm{CP}$ in a rotated squareshaped slot placed on top metallic lid. Parametric simulation studies demonstrate that a difference between both arms length of approximately $\lambda / 4$ leads to high-purity $C P$ within a relatively broad bandwidth. A square-shaped slot antenna is manufactured and experimentally analyzed. A broadband matching with a reflection coefficient magnitude below $-10 \mathrm{~dB}$ $(\mathrm{S} 11<-10 \mathrm{~dB})$ is achieved from 60.5 to $69.3 \mathrm{GHz}$. Applying the axial ratio criterion ( $A R<3 \mathrm{~dB})$ the bandwidth in $C P$ is $10.74 \%$, with respect to the central frequency. The maximum gain at broadside is $5.49 \mathrm{~dB}$ at $66.8 \mathrm{GHz}$.
\end{abstract}

Index Terms-antennas, gap waveguide, circular polarization, broadband.

\section{INTRODUCTION}

The $60 \mathrm{GHz}$ frequency band is nowadays attracting a lot of attention, due to the benefits it offers in many upcoming applications. One of the main drivers is the implementation of $5 \mathrm{G}$ mobile communications that is generating great expectations in telecommunications technology, as it holds the promise to address the Internet of Things (IoT) paradigm. The envisioned systems demand high data rate communications with a capacity unattainable in the classical microwave band, a fact that is pushing the limits towards higher frequencies in the quest of complying with the wider bandwidth (BW) required. Therefore, devising systems and operating at higher frequencies becomes a necessity and in this context antennas can be seen as key devices to provide wireless connection between the foreseen systems.

One of the main problems that have hindered the full development of millimeter-wave systems is the high loss of traditional feeding systems like waveguides and microstrip lines that suffer from increasing loss as the frequency grows. Hence, new technological variants specifically designed at millimeter-waves must be found. In recent years, gap waveguide $(\mathrm{GW})$ technology has arisen as an interesting alternative, improving considerably traditional solutions as it has low loss at millimeter-waves, does not require electric contact and is easily adaptable to flat surfaces [1], [2]. [3]. In addition, GW devices has a lower manufacturing cost with respect to traditional hollow waveguides, since the tolerances are coarser.

GW technology has three fundamental variants: Groove Gap Waveguide (GGW) [4], Ridge Gap Waveguide (RGW) [5]-[7] and Microstrip Gap Waveguide (MGW) [8]. Amongst them, the one with highest loss is MGW as it needs a dielectric substrate that introduced dielectric loss. Therefore, the best candidates for low-loss millimeter-wave devices are GGW and RGW, being the latter the solution adopted in this work.

In addition to reducing loss of feeding systems, it is equally important to minimize path loss. In this regard, circular polarization (CP) has several advantages over linear polarization: $\mathrm{CP}$ does not require polarization alignment between the transmitter and the receiver and is more robust against multipath effects [9]. There are several examples in the literature of CP antennas using GW technology. In [10], a $\mathrm{CP}$ antenna made of an array of rotated rectangular slots was designed in the band of $75-80 \mathrm{GHz}$. The proposed waveguide slot antenna array in [11], [12], have a good performance, they use RGW and GGW respectively in the Ka-band for generating CP. It is noteworthy that all these designs have a $\mathrm{BW}$ below $5 \mathrm{GHz}$ and, in addition, are relatively complex to manufacture.

In this work, a novel way to generate $\mathrm{CP}$ using $\mathrm{RGW}$ technology is presented. It is based on a simple topology able to excite two orthogonal modes, which combined with the required phase shift and the same amplitude produce $\mathrm{CP}$ at the output. The main objective is to achieve the widest possible BW with medium gain, in order to offer a competitive antenna for applications in the $60 \mathrm{GHz}$ band.

\section{ANTENNA CONFIGURATION AND DESIGN}

All the numerical results in this work were obtained using the commercial simulator CST Microwave Studio ${ }^{\circledR}$. Fig 1 shows the initial design.

(C) 2020 IEEE. Personal use of this material is permitted. Permission from IEEE must be obtained for all other uses, in any current or future media, including reprinting/republishing this material for advertising or promotional purposes, creating new collective works, for resale or redistribution to servers or lists, or reuse of any copyrighted component of this work in other work 


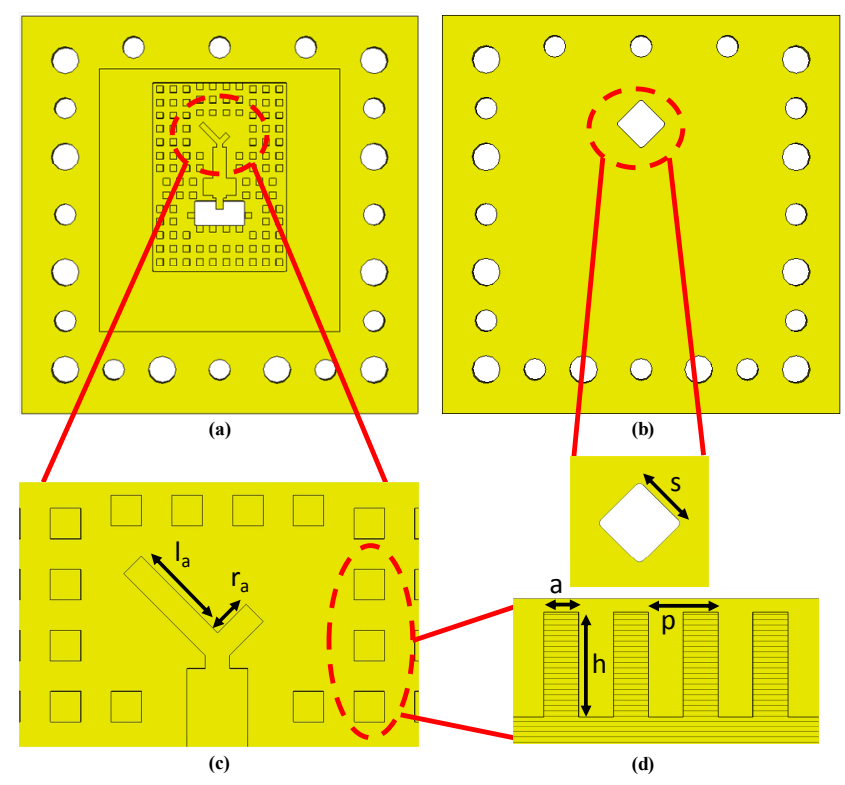

Fig 1. Schematic of the antennas (a) feeding face (b) radiation face. Detailed view of (c) left arm $\left(l_{a}\right)$ and right arm $\left(r_{a}\right)$ (d) square-shape slot $(s)$ and pines.

The antenna is optimized to work in the V-band of the millimeter-wave range and its design is fully metallic (aluminum) to minimize loss. The antenna is fed from the bottom with a WR-15 waveguide, which couples the wave to the RGW through matching section shown in Fig. 1(a). This feeding system ends in two perpendicular arms of different lengths, which excite a rotated square-shaped aperture on the upper plate, Fig. 1(b). The wave is guided by the ridge and the periodic metallic pin structure depicted in Fig. 1(d), which is equivalent to a high-impedance surface

The two perpendicular arms mentioned above (shown in Fig. 1(c)) in combination with the square-shape slot are responsible for the generation of $\mathrm{CP}$. Theoretically, a difference in length of $\lambda / 4$ between both arms should excite two orthogonal modes, which combined induce a circularly polarized wave at the output.

A parametric study was performed to find the optimum length difference between the ending arms. The effect of the variation of the length of both arms $\left(l_{a}\right.$ and $\left.r_{a}\right)$ and the slot length $s$ on the antenna matching and the axial ratio is shown in Fig. 2. To ensure a good operation the first condition that must be fulfilled is having a reflection coefficient below $-10 \mathrm{~dB}\left(\mathrm{~S}_{11}<-10 \mathrm{~dB}\right)$ in the operation band. In the cases studied, this criterion in the BW of interest is achieved when the difference between arms $\left(l_{a}-r_{a}\right)$ was $\lambda / 4$ (blue and green curve Fig. 2(a)). Next, to have a good CP purity, the axial ratio criterion should be below $3 \mathrm{~dB}$. Fig. 2(b) shows that when the arms length difference is approximately $\lambda / 4$, the AR curve is below $3 \mathrm{~dB}$. In those cases, the CP BW (defined as the BW where $A R<3 \mathrm{~dB}$ ) is the best of all the studied cases (blue and green curves). Finally, the BW of the antenna is given by the overlap of both the matching $\mathrm{BW}$ and the $\mathrm{CP}$ BW: in the case the blue curve goes from 60.3 to $73.7 \mathrm{GHz}$ $(>13 \mathrm{GHz})$ and the green curve from 59 to $68.9 \mathrm{GHz}$ $(>9 \mathrm{GHz})$.
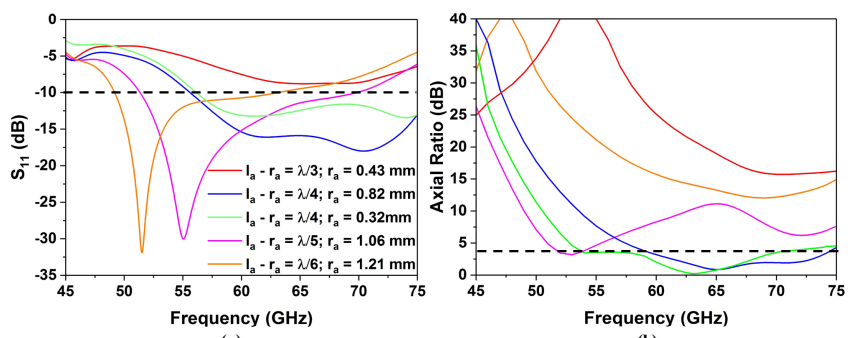

(a)

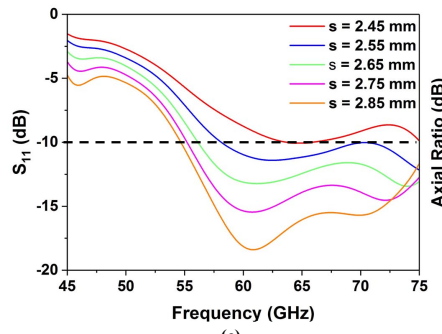

(c)

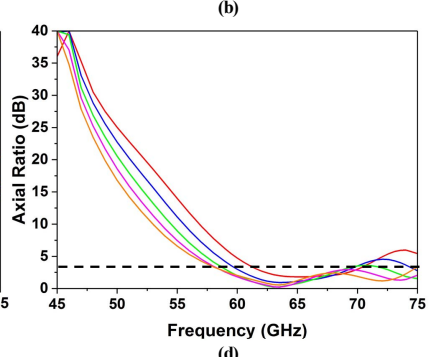

(d)

Fig. 2. Parametric simulations of both arms $\left(l_{a}\right.$ and $r_{a}$ ) (a) $\mathrm{S}_{11}$ (b) Axial ratio. Parametric simulations of square-shape slot (s) (c) $\mathrm{S}_{11}$ (d) Axial

A second parametric study considering the influence of the square-shape slot in the CP generation is realized. In this case, the arms values were kept constant and equal to $l_{a}=2$ $\mathrm{mm}$ and $r_{a}=0.82 \mathrm{~mm}$ (blue curve Fig. 2(a), (b)). Fig. 2(c) shows that the square side $s$, has a strong influence on the reflection coefficient. For small values of $s$, there is impedance mismatch within all the considered BW. However, when $s$ increases, the antenna impedance matching improves noticeably. Fig. 2(d) shows the effect of $s$ on the AR. Although the polarization purity increases with $s$, it is observed that in all cases we have acceptable values of $\mathrm{AR}<3 \mathrm{~dB}$ in the $\mathrm{BW}$ of interest. These results demonstrate that $\mathrm{CP}$ critically depends on the length difference of the ending arms. The simulated radiation efficiency of the antenna in all cases studied is above $95 \%$.

Finally, to fine-tune all the antenna dimensions a truth region framework optimization method (available in the

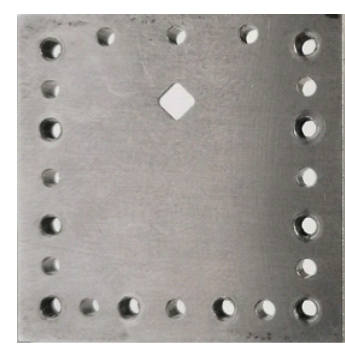

(a)

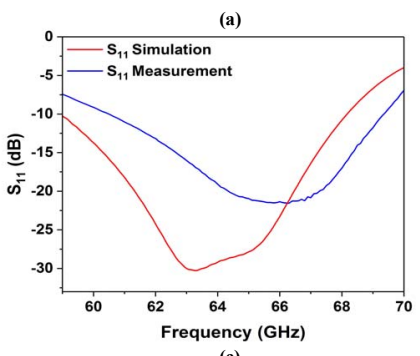

(c)

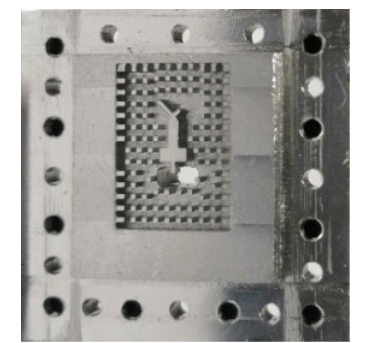

(b)

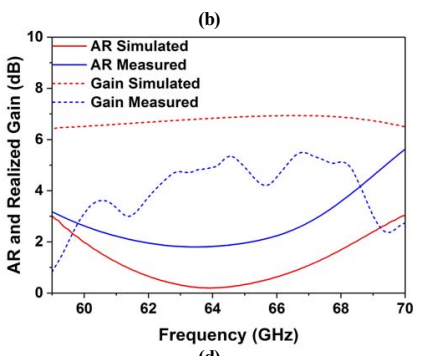

(d)
Fig. 3. Photographs showing a view of: (a) radiation face (b) Feeding face. Simulation and experimental results: (c) reflection coefficient (d) Axial Ratio and Realized Gain at broadside $\varphi=0^{\circ}$ and $\theta=0^{\circ}$. 
simulator) was applied. After these optimization the next parameters were obtained: $l_{a}=1.97 \mathrm{~mm}, r_{a}=0.86 \mathrm{~mm}, s=$ $2.66 \mathrm{~mm}$. Next, a prototype was manufactured using a Computer Numerical Control (CNC) milling machine to corroborate experimentally the numerical findings.

Fig. 3(a) and (b) show photographs of the manufactured antenna. A PNA network analyzer E8361C (Agilent Technologies) was used in the frequency range from 59 to $70 \mathrm{GHz}$ and the frequency span was discretized with steps of $50 \mathrm{MHz}$. The CP was measured with a linearly polarized horn antenna (Mi-Wave 261). To obtain the CP the test antenna was rotated to measure orthogonal linear polarization and from recorded data, the $\mathrm{CP}$ characteristics (gain, axial ratio, etc.) were calculated. Fig. 3(c) shows the reflection coefficient. The simulation and experimental results have a good agreement with a BW from 60.5 to 69.3 GHz. The AR and realized gain is shown in Fig. 3(d). From there, we find that the CP BW $(\mathrm{AR}<3 \mathrm{~dB})$ goes from 59.2 to $67.4 \mathrm{GHz}$ again with a good agreement with the simulation results. From these result, the practical operation BW is approximately $6.9 \mathrm{GHz}, 60.5$ to $67.4 \mathrm{GHz}$. The realized gain (blue curve) has a maximum of $5.49 \mathrm{~dB}$ at $66.8 \mathrm{GHz}$.

\section{CONCLUISION}

In this work, a CP antenna based on RGW technology operating in the V-band of the millimeter-wave spectrum is presented. By using two arms of different lengths in the feeding system it is feasible to CP in a simple way, without the need of implementing an array along with a complex feeding network. A practical operation BW approximately 6.9 $\mathrm{GHz}$ was achieved in the band from 60.5 to $67.4 \mathrm{GHz}$, a result that represents a fractional BW of $10.74 \%$. This antenna could open new avenues in technological applications related to future $5 \mathrm{G}$ applications in the context of the IoT paradigm.

\section{ACKNOWLEDGMENT}

This research was funded by the Spanish Ministerio de Ciencia, Innovación y Universidades, Project RTI2018094475-B-I00 (MCIU/AEI/FEDER, UE)

\section{REFERENCES}

[1] J. Yang, P.-S. Kildal, E. Alfonso, and H. Raza, "Resemblance between gap waveguides and hollow waveguides," IET Microwaves, Antennas Propag., vol. 7, no. 15, pp. 1221-1227, Dec. 2013.

[2] P.-S. Kildal, E. Alfonso, A. Valero-Nogueira, and E. Rajo-Iglesias, "Local Metamaterial-Based Waveguides in Gaps Between Parallel Metal Plates," IEEE Antennas Wirel. Propag. Lett., vol. 8, pp. 8487, 2009 .

[3] J. Hirokawa and M. Zhang, Handbook of Antenna Technologies. Singapore: Springer Singapore, 2014.

[4] A. Berenguer, V. Fusco, D. E. Zelenchuk, D. Sanchez-Escuderos, M. Baquero-Escudero, and V. E. Boria-Esbert, "Propagation Characteristics of Groove Gap Waveguide Below and Above Cutoff," IEEE Trans. Microw. Theory Tech., vol. 64, no. 1, pp. 2736, Jan. 2016.

[5] A. U. Zaman and P. Kildal, "Wide-Band Slot Antenna Arrays With Single-Layer Corporate-Feed Network in Ridge Gap Waveguide Technology," IEEE Trans. Antennas Propag., vol. 62, no. 6, pp. 2992-3001, 2014.
[6] D. Zarifi, A. Farahbakhsh, A. U. Zaman, and P.-S. Kildal, "Design and Fabrication of a High-Gain $60-\mathrm{GHz}$ Corrugated Slot Antenna Array With Ridge Gap Waveguide Distribution Layer," IEEE Trans. Antennas Propag., vol. 64, no. 7, pp. 2905-2913, Jul. 2016.

[7] J. Liu, A. Vosoogh, A. U. Zaman, and J. Yang, "A Slot Array Antenna with Single-Layered Corporate-Feed based on Ridge Gap Waveguide in the 60-GHz Band," IEEE Trans. Antennas Propag., vol. PP, no. c, pp. 1-1, 2018.

[8] J. Liu, A. Vosoogh, A. U. Zaman, and J. Yang, "Design and Fabrication of a High-Gain $60-\mathrm{GHz}$ Cavity-Backed Slot Antenna Array Fed by Inverted Microstrip Gap Waveguide," IEEE Trans. Antennas Propag., vol. 65, no. 4, pp. 2117-2122, Apr. 2017.

[9] T. Manabe, K. Sato, H. Masuzawa, K. Taira, T. Ihara, Y. Kasashima and K. Yamaki , "Polarization dependence of multipath propagation and high-speed transmission characteristics of indoor millimeterwave channel at $60 \mathrm{GHz}$," IEEE Trans. Veh. Technol., vol. 44, no. 2, pp. 268-274, May 1995.

[10] J. Xi, B. Cao, H. Wang, and Y. Huang, "A novel $77 \mathrm{GHz}$ circular polarization slot antenna using ridge gap waveguide technology," in 2015 Asia-Pacific Microwave Conference (APMC), 2015, pp. 1-3.

[11] T. Li and F. Fan, "Design of ka-band $2 \times 2$ circular polarization slot antenna array fed by ridge gap waveguide," in 2017 Sixth AsiaPacific Conference on Antennas and Propagation (APCAP), 2017, pp. 1-3.

[12] M. Ferrando-Rocher, J. I. Herranz-Herruzo, A. Valero-Nogueira, and A. Vila-Jimenez, "Single-Layer Circularly-Polarized Ka-Band Antenna Using Gap Waveguide Technology," IEEE Trans. Antennas Propag., vol. 66, no. 8, pp. 3837-3845, Aug. 2018. 